\title{
Supporting information \\ Untargeted Proteomics-Based Profiling for the Identification of Novel Processing-induced Protein Modifications in Milk
}

Jasmin Meltretter, ${ }^{\dagger, \S}$ Johannes Wüst, ${ }^{\dagger}, \S$ Daniel Dittrich, ${ }^{\dagger, \S}$ Johannes Lach, ${ }^{\ddagger}$ Jonas Ludwig, ${ }^{\sharp}$ Jutta Eichler, ${ }^{\ddagger}$ Monika Pischetsrieder ${ }^{\dagger}, *$

$\dagger$ Department of Chemistry and Pharmacy, Food Chemistry, Emil Fischer Center, Friedrich-Alexander-Universität Erlangen-Nürnberg (FAU), Nikolaus-Fiebiger-Str. 10, 91058 Erlangen, Germany

* Department of Chemistry and Pharmacy, Medicinal Chemistry, Emil Fischer Center, Friedrich-Alexander-Universität Erlangen-Nürnberg (FAU), Nikolaus-Fiebiger-Str. 10, 91058 Erlangen, Germany

$\S$ The authors contributed equally to this work.

* Corresponding author: Prof. Dr. Monika Pischetsrieder, Henriette Schmidt-Burkhardt Chair of Food Chemistry, Department of Chemistry and Pharmacy, Emil Fischer Center, FriedrichAlexander-Universität Erlangen-Nürnberg (FAU), Nikolaus-Fiebiger-Str. 10, 91058 Erlangen, Germany, Tel. +49-9131-8565592, E-mail: monika.pischetsrieder@fau.de

\section{Table of Contents}

Table S1. Transitions and Optimized Settings for the MRM Analysis of the Newly Identified

Peptide Modifications in Milk.................................................... S-2 
Table S1. Transitions and Optimized Settings for the MRM Analysis of the Newly Identified Peptide Modifications in Milk.

Precursor ions including charge states (z), corresponding quantifier (quant.) and qualifier (quali.) ions with collision energies (CE) and declustering potentials (DP) are given.

\begin{tabular}{|c|c|c|c|c|c|c|c|c|c|}
\hline $\mathrm{AA}^{\mathrm{a}}$ & Sequence & Modification & Localization & $\begin{array}{l}\text { Precursor } \\
\text { ion } m / z\end{array}$ & $z$ & $\begin{array}{l}\text { Fragment } \\
\text { ion } m / z\end{array}$ & $\begin{array}{l}\mathrm{CE} \\
{[\mathrm{eV}]}\end{array}$ & $\begin{array}{l}\text { DP } \\
{[\mathrm{V}]}\end{array}$ & Info \\
\hline & & & & 615.8 & 2 & 893.5 & 26 & 70 & Quant. \\
\hline \multirow[t]{3}{*}{$1-11$} & LIVTQTMKGLD & 4-Imidazolidinone & N-Terminus & 615.8 & 2 & 664.3 & 25 & 70 & Quali. \\
\hline & & & & 615.8 & 2 & 984.7 & 25 & 70 & Quali. \\
\hline & & & & 516.3 & 2 & 700.3 & 30 & 70 & Quant. \\
\hline \multirow[t]{3}{*}{$66-74$} & CAQKKIIAE & N-Formyllysine & K70 & 516.3 & 2 & 813.4 & 25 & 50 & Quali. \\
\hline & & & & 516.3 & 2 & 587.3 & 35 & 50 & Quali. \\
\hline & & & & 790.2 & 1 & 643.4 & 35 & 50 & Quant. \\
\hline \multirow[t]{3}{*}{$128-134$} & ViDDEALE & Isomerization & D129 & 790.2 & 1 & 459.2 & 35 & 70 & Quali. \\
\hline & & & & 790.2 & 1 & 530.3 & 35 & 70 & Quali. \\
\hline & & & & 790.2 & 1 & 643.4 & 38 & 70 & Quant. \\
\hline \multirow[t]{2}{*}{$128-134$} & VDiDEALE & Isomerization & D130 & 790.2 & 1 & 459.2 & 38 & 70 & Quali. \\
\hline & & & & 790.2 & 1 & 530.3 & 38 & 70 & Quali. \\
\hline
\end{tabular}

${ }^{\mathrm{a}} \mathrm{AA}$, amino acid 